\title{
State-of-the-Art of Serum Total Calcium Measurement as Investigated by Split-Sample Measurement with an Ion Chromatography Candidate Reference Method
}

\author{
Jean E. Van Nuwenborg, Dietmar Stöckl and Linda M. Thienpont
}

Laboratorium voor Analytische Chemie, Faculteit van de Farmaceutische Wetenschappen, Universiteit Gent, Gent, Belgium

Summary: We evaluated bias and inaccuracy of four frequently used routine test systems for serum total calcium, using a candidate reference method based on ion chromatography. The mean biases and $95 \%$ confidence intervals that we observed were $0.0 \pm 0.59 \%$ for Johnson \& Johnson arsenazo(III), $1.3 \pm 0.62 \%$ for Beckman arsenazo(III), $-0.4 \pm 0.44 \%$ for Beckman applying ion selective electrode measurement after sample dilution, and $-1.9 \% \pm 0.42 \%$ for Boehringer $o$-cresolphthalein. The inaccuracy of all test systems was usually $<4.7 \%$ (calculated as deviation of singlicates from ion chromatography). Both bias and inaccuracy are discussed in the light of specifications set by expert groups or derived from the biological variation of serum total calcium. The study revealed that the intrinsic quality of commonly used test systems for serum total calcium satisfies even some of the more stringent criteria for method bias and inaccuracy.

\section{Introduction}

Measurement of serum total calcium is still considered a challenge for the routine laboratory, in particular when analytical specifications are applied that have been derived from the biological variation of the analyte (1). We therefore investigated the state-of-the-art of the accuracy and specificity of routine serum total calcium measurements. Four frequently used routine test systems for serum total calcium were evaluated, using a recently developed candidate reference method based on ion chromatography $(2,3)$, applying split sample measurement of a panel of patient samples. The main emphasis of the study was assessment of the overall bias of the test systems and their sensitivity to common sample matrices. Quality control sera often exhibit matrix effects $(4,5)$ and are hence not always representative for the real test system performance. Therefore quality control materials were not included in the study.

Here, we present the observed deviations of the routine test systems from the reference method, applying different specifications for analytical bias and inaccuracy $(6-10)$.

\section{Materials and Methods}

Operation of the routine test systems was the responsibility of the respective manufacturers or distributors. The following methods were investigated:

(i) the arsenazo-III method from Johnson \& Johnson Clinical Diagnostics (Rochester, NY, USA), performed in the Johnson \& John- son application laboratory at Illkirch (France) on a Vitros 250 analyzer;

(ii) the arsenazo-III method and

(iii) the indirect ion selective electrode (ISE) method from Beckman Instruments (Brea, CA, USA), performed at the site of the Belgian distributor Analis (Namur, Belgium) on a Synchron CX4 and a CX3 Delta analyzer, respectively; and

(iv) the o-cresolphthalein method from Boehringer Mannheim (Tutzing, Germany), performed in their application laboratory on a Hitachi 717 analyzer.

The patient panel used for the study was kindly provided by the Hospital "Institut Moderne" of Gent (Belgium). The samples were treated in accordance with the ethical standards of the Committee for Medical Ethics of the University of Gent. The panel consisted of 88 randomly collected samples. All had been ordered by physicians for total calcium analysis. They were stored at $-80^{\circ} \mathrm{C}$ before their shipment in dry ice to the respective application laboratories. Because of the focus of our study on test system calibration and behaviour with common samples, no efforts were made to collect samples with calcium concentrations far beyond the reference limits or samples that might contain specific interferences.

Measurements by the routine systems were performed in triplicate within one run and under rigorous internal quality control. Each manufacturer used his own internal quality control samples. They were placed at the beginning of the run $(n=9)$, each tenth sample $(n=3)$, and at the end $(n=9)$.

The number of results reported back to us were 88 for Boehringer and Beckman indirect ISE, 82 for Johnson \& Johnson, and 59 for Beckman arsenazo. The reduced number in the latter case was due to limited sample volume. Measurements by ion chromatography $(2,3)$ were performed in batches of 9-10 samples over a period of 9 days. This measurement scheme was necessary because of the relatively long chromatographic runs. Also the ion chromatography measurements were performed in triplicate, but each aliquot was independently prepared. The internal quality control samples for ion chromatography were the certified reference materials (CRMs) 303 and 304 from the Bureau Communautaire de Référence (BCR, 
Brussels, Belgium). They were measured at the start, in the middle and at the end of each run.

\section{Results and Discussion}

\section{Internal quality control}

In order to obtain results that were representative for the respective test systems, the whole study was performed in the application laboratories of the respective manufacturers or distributors under strict internal quality control, measuring at least two control samples in blocks at the start, in the middle and at the end of each run. The inaccuracy limit of the mean of the internal quality control measurements for each of the control samples was set at $1.5 \%$. The respective target values were method-dependent assigned values for both Beckman systems, and reference method values for the Boehringer test system. Johnson \& Johnson uses target ranges determined by measurement of their control samples by numerous laboratories using their routine kit. We used the mean of this range as the target value.

The following mean deviations from the respective target values were found: Johnson \& Johnson $-2.2 \%$ (at $2.30 \mathrm{mmol} / \mathrm{l}$ ) and $0.8 \%$ (at $2.90 \mathrm{mmol} / \mathrm{l}$ ); Beckman arsenazo/indirect ISE $0.3 \% /-0.6 \%$ (at $2.28 / 2.33$ $\mathrm{mmol} / \mathrm{l}$ ), $-0.4 \% /-1.0 \%$ (at $2.83 / 2.85 \mathrm{mmol} / \mathrm{l}$ ), and $-1.0 \% /-1.2 \%$ (at $3.45 / 3.43 \mathrm{mmol} / \mathrm{l}$ ); Boehringer $-0.4 \%$ (at $2.19 \mathrm{mmol} / \mathrm{l}$ ), $-0.7 \%$ (at $2.65 \mathrm{mmol} / \mathrm{l}$ ), and $\pm 0.0 \%$ (at $3.32 \mathrm{mmol} / \mathrm{l}$ ). The maximal allowable inaccuracy of the individual internal quality control blocks ( 9 measurements at the start and the end of the run; 3 measurements after each tenth patient sample) was calculated from the maximal allowable bias $(1.5 \%)$, the mean $\mathrm{CV}$ obtained for that specific control serum and the $95 \%$ confidence interval. As an example we cite the Beckman arsenazo measurements with a mean $\mathrm{CV}$ of $0.7 \%$ (for all three control samples). The maximal allowable deviation from the target should then be $3.2 \%$ for the triplicates and $2.0 \%$ for the 9-fold measurements. The deviations for all individual internal quality control runs were within these calculated limits. For Johnson \& Johnson, the measured value (global mean and individual mean per internal quality control block) always lays in the control range, but the deviation from our fixed target value was higher than $1.5 \%$ or the value calculated as explained above. Considering the fact that Johnson \& Johnson do not use a target value, they considered the quality control results as representative for the system. The mean CV (CV range) of the quality control runs for each of the test systems were: $0.6 \%(0.2$ to $0.9 \%$ ) for Johnson \& Johnson, $0.7 \%(0.2$ to $1.1 \%)$ for Beckman arsenazo, $0.4 \%$ (0.1 to $0.9 \%)$ for Beckman ISE, and $0.5 \%$ ( 0 to $0.8 \%$ ) for Boehringer. The recommended within-day CV values were $1.5 \%$ for Johnson \& Johnson and $1 \%$ for the both Beckman and the Boehringer systems.
The mean deviations and between-day standard deviations for the ion chromatographic method were 0.2 $\pm 0.8 \%(\mathrm{CRM} 303,2.47 \mathrm{mmol} / \mathrm{l})$ and $0.6 \pm 0.5 \%$ (CRM 304, $2.20 \mathrm{mmol} / \mathrm{l}$ ).

From these data, we conclude that all test systems were under adequate control during the study.

\section{Evaluation of routine method bias}

The results of the method evaluations are presented as bias plots (see fig. 1, each data point was calculated from the mean of within-run triplicates) (note: linear regression was not applied because of the narrow concentration range of the samples). The following outlying samples (routine results differing more than $15 \%$ from ion chromatography) were excluded from the further calculations: samples $67(-20.9 \%)$ and 75 $(-18.2 \%)$ for Johnson \& Johnson and sample 54 $(-17.0 \%)$ for Boehringer. Further, 4 samples had to be excluded because of internal quality control problems of ion chromatography in one run (repetition was not possible because of limited sample volume). From the data in figure 1, mean biases and $95 \%$ confidence intervals were calculated: $0.0 \pm 0.59 \%$ for Johnson \& Johnson, $1.3 \pm 0.62 \%$ for Beckman arsenazo, -0.4 $\pm 0.44 \%$ for Beckman indirect ISE, and -1.9 $\pm 0.42 \%$ for Boehringer. For the following interpretation, it should be noted that a bias in the order of -0.6 to $+1.1 \%$ has to be taken into account for the ion chromatographic method itself (see internal quality control data of the ion chromatographic method). According to the most stringent model of Gowans et al. (6), which is based on the biological variation of serum total calcium, routine methods should have values for bias $<0.7 \%$. However, since the bias of the ion chromatography reference method already exceeded this limit, it was not possible to assess whether the routine test systems fulfilled the specifications set by Gowans. According to the model of Harris (7), using the specification that routine method bias should be $<1 / 8$ of the reference range, a bias limit of $1.8 \%$ would be calculated. Considering the possible bias of -0.6 to $+1.1 \%$ of the ion chromatographic method, the limits for bias in this study would be $-2.9 \%$ and $+2.4 \%$ for the Harris model. With the above calculated data for bias (including the $95 \%$ confidence interval), it can be concluded that all four routine test systems satisfy the latter criterion for bias. The low values for the $95 \%$ confidence intervals of the biases additionally indicate that the test systems were not influenced by the common patient matrices.

\section{Evaluation of routine method inaccuracy}

Routine method inaccuracy was evaluated by calculation of the deviation of single routine results from the mean 

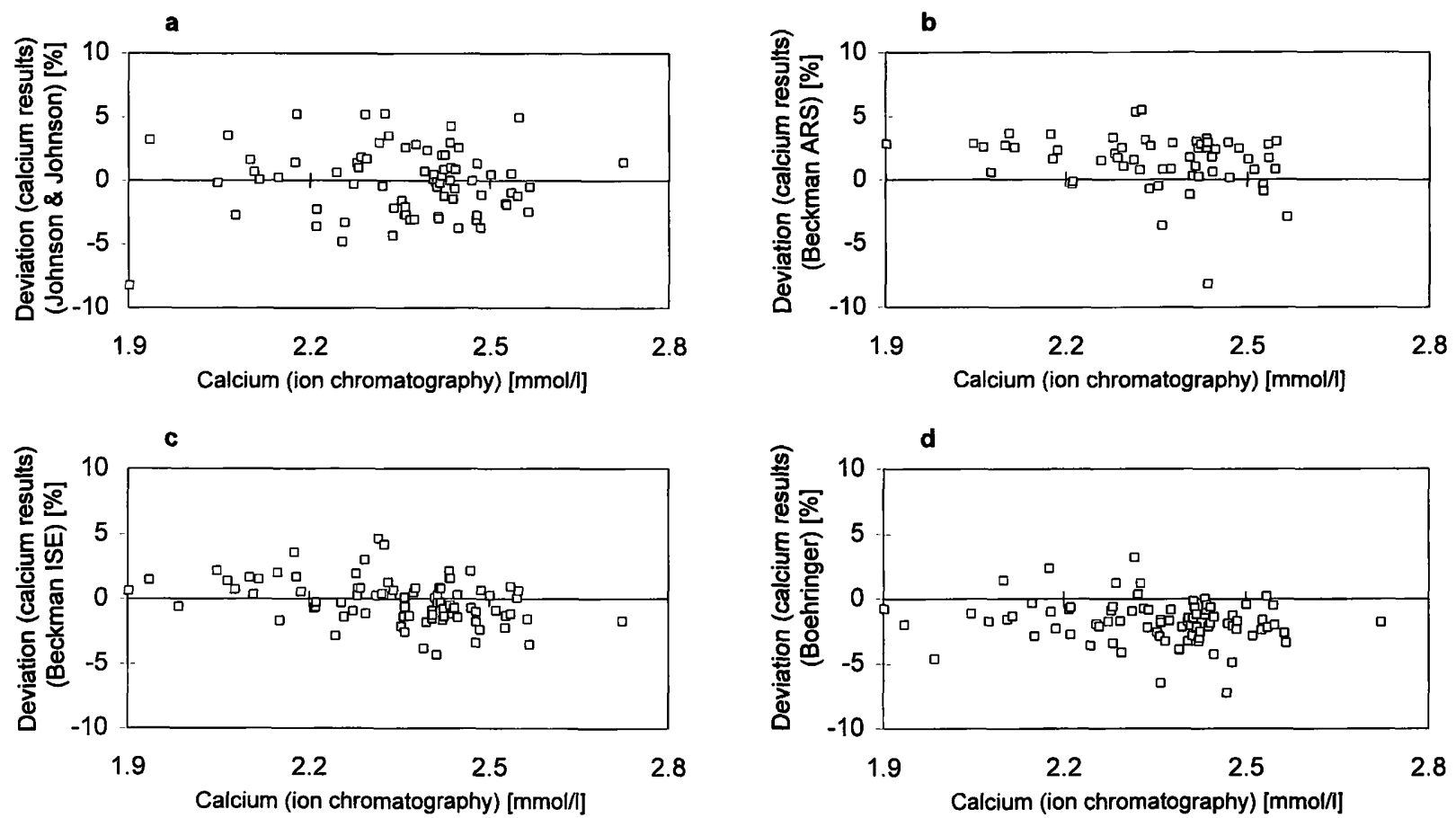

Fig. 1 Bias plots of the routine test system results vs those obtained by ion chromatography: Johnson \& Johnson arsenazo(III) (a), Beckman arsenazo(III) (b), Beckman ISE (c), and Boehringer

$o$-cresolphthalein (d) (Note: each data point was calculated from the mean of within-run triplicates).

of triplicates obtained with the ion chromatographic method. These deviations are assessed against various criteria for total error. Among them are the total error criteria of $10 \%$ as applied in the German (8) and American (9) external quality assessment surveys, and the total error criterion as expressed by Tonks (10), being $1 / 4$ of the reference range, resulting in a limit of $3.7 \%$. However, those criteria have to be expanded in our case by the inaccuracy of the ion chromatographic method which lies in the order of $1 \%$ (see above), resulting in limits of 11 and $4.7 \%$ for the deviation of singlicates. It should be noted further that these limits usually should be satisfied in $95 \%$ of the measurements performed. Deviations $>11 \%$ were observed for none of the test systems (except for outliers as already indicated), demonstrating that all test systems are able to satisfy limits of total error applied in external quality assessment. The total error limit, according to the model of Tonks, was exceeded in $6.4 \%$ of the samples for Johnson \& Johnson, in $3.5 \%$ of the samples for Beckman arsenazo, in $3.6 \%$ of the samples for Beckman indirect ISE, and in $4.8 \%$ of the samples for Boehringer. Three of the systems tested also satisfied this more stringent limit, and only the Johnson \& Johnson test system exceeded the limit in slightly more than $5 \%$ of the cases. It should be noted here that the low values for total error stem from the excellent within-run imprecision of the test systems. The mean within-run CV (calculated from the triplicates) was in the order of $0.5 \%$ for Johnson \& Johnson, Beckman ISE, and Boehringer. Only the Beckman arsenazo method showed a slightly increased within-run $\mathrm{CV}$, namely $0.8 \%$.

\section{Conclusion}

The study revealed that the intrinsic quality of commonly used test systems for serum total calcium satisfies even some of the more stringent criteria for method bias and inaccuracy. However, the study was undertaken under within-run and rigorous internal quality control conditions, and in the application laboratories of the respective manufacturers. The same quality of performance cannot be expected in the routine laboratory. However, a similar investigation in the routine laboratory would need a different experimental design, e.g., larger amounts of samples sent to at least 5 different laboratories per test system, and treatment of the samples as normal routine samples. We therefore conclude that routine measurements of serum total calcium are still a challenge for the average routine laboratory. This is particularly true for appropriate installation of a test system as well as the efforts that have to be investigated for internal quality control. In particular, laboratories should use stringent internal quality control criteria and control materials with highly accurate target values, and should take special care to reduce between-run variability.

\section{Acknowledgements}

This research was supported by the Research Fund of the University of Gent under Grant 01116692. 


\section{References}

1. Fraser CG, Hyltoft Petersen P, Ricos C, Haeckel R. Proposed quality specifications for the imprecision and inaccuracy of analytical systems for clinical chemistry. Eur J Clin Chem Clin Biochem 1992; 30:311-7.

2. Thienpont LM, Van Nuwenborg JE, Stöckl D. Ion chromatography as potential reference methodology for the determination of total calcium and magnesium in human serum. Anal Chem 1994; 66:2404-8.

3. Thienpont LM, Van Nuwenborg JE, Reinauer H, Stöckl D. Validation of candidate reference methods based on ion chromatography for the determination of total sodium, potassium, calcium, and magnesium in serum through comparison with flame atomic emission and absorption spectrometry. Clin Biochem 1996; 29:501-8.

4. Külpmann WR, Buchholz R, Dyrssen C, Ruschke D. A comparison of reference method values for calcium, lithium and magnesium with method-dependent assigned values. J Clin Chem Clin Biochem 1989; 27:631-7.

5. Külpmann WR, Maibaum $P$, Sonntag $O$. Analyses with the KODAK-Ektachem. Accuracy control using reference method values and the influence of protein concentration. Part I. Electrolytes. J Clin Chem Clin Biochem 1990; 28:825-33.

6. Gowans EMS, Hyltoft Petersen P, Blaabjerg O, Horder M. Analytical goals for the acceptance of common reference intervals for laboratories throughout a geographical area. Scand J Clin Lab Invest 1988; 48:757-64.

7. Harris EK. Proposed goals for analytical precision and accuracy in single point testing. Arch Pathol Lab Med 1988; $112: 416-20$.

8. Tonks DB. A study of accuracy and precision of clinical chemistry determinations in 170 Canadian laboratories. Clin Chem 1963; 9:217-33.

9. Richtlinien der Bundesärztekammer zur Qualitätssicherung in medizinischen Laboratorien. Dtsch Ärztebl 1988; 85:A699A712.

10. Department of Health and Human Services. Medicare, Medicaid and CLIA programs; regulations implementing the Clinical Laboratory Improvement Amendments of 1988 (CLIA). Fed Regist, 1992 Feb 28; 57:7002-186.

Received October 25/December 20, 1996

Corresponding author: Professor Dr. Linda Thienpont, Laboratorium voor Analytische Chemie, Faculteit Farm.

Wetenschappen, Universiteit Gent, Harelbekestraat 72, B-9000 Gent, Belgium

FAX: +32-9-264 81 98, E-MAIL: Linda.Thienpont@rug.ac.be 